\title{
LOCAL EXPANSIONS AND ACCRETIVE MAPPINGS
}

\author{
W.A. KIRK \\ Department of Mathematics \\ The University of Iowa \\ Iowa City, Iowa 52242 U.S.A. \\ (Received February 14, 1983 and in revised form March 24, 1983)
}

ABSTRACT. Let $\mathrm{X}$ and $\mathrm{Y}$ be complete metric spaces with $\mathrm{Y}$ metrically convex, let $D \subset X$ be open, fis $u_{0} \in X$, and let $d(u)=d\left(u_{0}, u\right)$ for all $u \in D$.

Let $f: X \longrightarrow 2^{Y}$ be a closed mapping which maps open subsets of $D$ onto open sets in $Y$, and suppose $f$ is locally expansive on $D$ in the sense that there exists a continuous nonincreasing function $c: \mathrm{R}^{+} \longrightarrow \mathrm{R}^{+}$with $\int^{+\infty} \mathrm{c}(\mathrm{s}) \mathrm{ds}=+\infty$ such that each point $x \in D$ has a neighborhood $N$ for which $\operatorname{dist}(f(u), f(v))$

$z c(\max \{d(u), d(v)\}) d(u, v)$ for all $u, v \in N$. Then, given $y \in Y$, it is shown that $y \in f(D)$ iff there exists $x_{0} \in D$ such that for $x \in X \backslash D$, dist $\left(y, f\left(x_{0}\right)\right)$

$\leq \operatorname{dist}(u, f(x))$. This result is then applied to the study of existence of zeros of (set-valued) locally strongly accretive and $\varnothing$-accretive mappings in Banach spaces.

KEY WORDS AND PHRASES. Local expansions, accretive mappings, nonexpansive mappings, fixed points, zeros.

1980 MATHEMATICS SUBJECT CLASSIFICATION CODES. Primary 47H10; Secondary $54 H 25$.

\section{INTRODUCTION}

This paper may be viewed as a sequel to that of Kirk and Schöneberg [1]. We first prove a general theorem for "local expansions" and we then apply this result in special settings to the study of the existence of zeros of the locally strongly accretive and $\phi$-accretive mappings. In the interest of attaining the generality readily offered by our techniques, we formulate our results for set-valued mappings even though some of our assumptions (e.g., continuity, as opposed to semicontinuity) might seem stringent for such mappings. The results themselves, however, represent 
extensions of those of [1] even in the point-valued case.

Results similar to those obtained here may be found in Ray and Walker [2] and in Torrejón [3]; however, the methods employed are different. Torrejon relies on differential inequalities, while Ray and Walker use the Brezis-Browder order principle to prove a refined version of the Caristi-Ekeland minimization principle, and this in turn is used to obtain, among other things, a Banach space version of the surjectivity part of our Theorem 2.1. On the other hand, Torrejon obtains our Theorem 2.1 under the assumptions that $X$ is a Banach space and $Y$ is a complete and metrically convex metric space. While it is likely that the methods of Ray-Walker and of Torrejon could be modified to attain the generality we obtain, our approach, which is a refinement of the argument of Kirk-Schöneberg [1], seems more direct and more in the spirit of the original work of Browder [4, \$4]. In particular, Browder uses an argument (cf. [4, Theorem 4.9]) roughly like the one we use below to show that a local expansion from a complete metric space $X$ to a metric space $Y$ is, under suitable connectedness hypotheses, actually a covering map of $X$ onto $Y$.

For the most part, we use standard notation. $\mathrm{B}(\mathrm{x} ; \mathrm{r})$ denotes the closed ball centered at a point $\mathrm{x}$ of a metric space with radius $\mathrm{r}>0$. We shall use $B(Y)$ and $\mathcal{C}(Y)$ to denote, respectively, the family of nonempty bounded closed subsets and the family of nonempty compact subsets of a metric space $Y$, and we assign to these families the usual Hausdorff metric (denoted by $H$ ). For a Banach space X, the mapping $J: X \longrightarrow 2^{X^{*}}$ denotes the usual normalized duality mapping:

$$
J(x)=\left\{j \in x^{*}:\|j\|=\|x\|,\langle x, j\rangle=\|x\|^{2}\right\}
$$

Also, for a subset $A$ of $X$, we use $|A|$ to denote $\inf \{\|x\|: x \in A\}$.

Finally, if $X$ and $Y$ are metric spaces, then a set-valued mapping $f: X \rightarrow 2^{X}$ is said to be closed if for $\left\{x_{n}\right\}$ in $x$, the conditions $x_{n} \rightarrow x, y_{n} \in T\left(x_{n}\right)$, and $\mathrm{y}_{\mathrm{n}} \rightarrow \mathrm{y}$ imply $\mathrm{y} \in \mathrm{T}(\mathrm{x})$.

2. A THEOREM ON LOCAL EXPANSIONS.

THEOREM 2.1. Let $(\mathrm{X}, \mathrm{d})$ be a complete metric space and ( $\mathrm{Y}, \mathrm{d})$ a rectifiably pathwise connected metric space with intrinsic metric $\ell$, let $D \subset X$ be open, fix $u_{0} \in X$, and let $d(u)=d\left(u_{0}, u\right), u \in D$. Let $f: X \rightarrow 2^{X}$ be a closed mapping which maps open subsets of $X$ onto open sets in $Y$, and suppose there exists a continuous 
nonincreasing function $c:[0, \infty) \rightarrow(0, \infty)$ with $\int^{+\infty} c(s) d s=+\infty$ such that each point $x \in D$ has a neighborhood $N$ for which

$$
\operatorname{dist}(f(u), f(v)) \geq c(\max \{d(u), d(v)\}) d(u, v)
$$

for all $u, v \in N$. Then, given $y \in Y$, the following are equivalent.

(a) $y \in f(D)$.

(b) There exists $x_{0} \in D$ such that for each $x \in X \backslash D$,

$$
\inf \left\{\ell(w, y): w \in f\left(x_{0}\right)\right\} \leq \inf \{\ell(w, y): w \in f(x)\} .
$$

In particular, if $D=X$, then $f$ is surjective.

PROOF. Since $(a) \Rightarrow(b)$ is trivial, we suppose (b) holds and show that the assumption $\mathrm{y} \notin \mathrm{f}(\mathrm{D})$ leads to a contradiction. For each $\mathrm{x} \in \mathrm{D}$, let

$$
\begin{aligned}
& r(x)=\sup \{r \in(0,1): B(x ; r) \subset D \text { and } \operatorname{dist}(f(u), f(v)) \\
& 2 c(\max \{d(u), d(v)\} d(u, v) \text { for all } u, v \in B(x ; r)\} .
\end{aligned}
$$

By assumption, $r(x)>0$ for each $x \in D$, and moreover if

$$
c=\inf \left\{c(d(u)): u \in B\left(x_{0} ; r\left(x_{0}\right) / 2\right)\right\} \text {, }
$$

then

$$
\epsilon=\operatorname{cr}\left(x_{0}\right) / 4>0
$$

We define a sequence $\left\{u_{n}\right\} \subset D$ as follows. Let $u_{1}=x_{0}, t_{1}=0$, and select $w_{1} \in f\left(u_{1}\right)$ and a path $\Gamma:[0,1] \rightarrow Y$ joining $w_{1}$ and $y \quad$ (with $\left.\Gamma(0)=w_{1}\right)$ such that the length, $\ell(\Gamma)$, of $\Gamma$ satisfies

$$
\ell(\Gamma) \leq \inf \left\{\ell(w, y): w \in f\left(x_{0}\right)\right\}+\epsilon .
$$

Let $t_{2}=\sup \left\{t \in[0,1]: \Gamma(t) \in f\left(B\left(u_{1} ; r\left(u_{1}\right) / 2\right)\right)\right\}$, let $\left\{s_{n}\right\} \subset[0,1]$ be such that $s_{n} t t_{2}$, and let $\Gamma\left(s_{n}\right) \in f\left(v_{n}\right)$ where $v_{n} \in B\left(u_{1} ; r\left(u_{1}\right) / 2\right), n=1,2, \cdots$ Since $\Gamma\left(s_{n}\right) \rightarrow \Gamma\left(t_{2}\right)$ and

$$
\mathrm{d}\left(\Gamma\left(\mathrm{s}_{\mathrm{n}}\right), \Gamma\left(\mathrm{s}_{\mathrm{m}}\right)\right) \geq \operatorname{dist}\left(f\left(\mathrm{v}_{\mathrm{n}}\right), f\left(\mathrm{v}_{\mathrm{m}}\right)\right) \geq \operatorname{cd}\left(\mathrm{v}_{\mathrm{n}}, \mathrm{v}_{\mathrm{m}}\right)
$$

it follows that $\left\{v_{n}\right\}$ converges to some point $v \in M$. Since $f$ is a closed mapping, $\Gamma\left(t_{2}\right) \in f(v)$. Also, since $y \in f(D), y \in f\left(B\left(u_{1} ; r\left(u_{1}\right) / 2\right)\right)$. In view of this, the fact that $f$ is open implies $v \in \partial B\left(u_{1} ; r\left(u_{1}\right) / 2\right)$. Now set $u_{2}=v$ and $w_{2}=\Gamma\left(t_{2}\right)$. Similarly, having defined $\left\{u_{i}\right\},\left\{t_{i}\right\}$, and $\left\{w_{i}\right\}$ for $i \in\{l, \cdots, n\}$, let

$$
t_{n+1}=\sup \left\{t \in[0,1]: \Gamma(t) \in f\left(B\left(u_{n} ; r\left(u_{n}\right) / 2\right)\right)\right\}
$$

and as above obtain $u_{n+1} \in \partial B\left(u_{n} ; r\left(u_{n}\right) / 2\right)$ for which $w_{n+1}=\Gamma\left(t_{n+1}\right) \in f\left(u_{n+1}\right)$. 
Thus, by induction, sequences $\left\{u_{n}\right\},\left\{t_{n}\right\}$, and $\left\{w_{n}\right\}$ exist satisfying for $n \in \mathbb{N}$,

$$
\begin{aligned}
& \text { (i) } t_{n+1}>t_{n} ; \\
& \text { (ii) } d\left(u_{n+1}, u_{n}\right)=r\left(u_{n}\right) / 2 ; \\
& \text { (iii) } c\left(\max \left\{d\left(u_{n}\right), d\left(u_{n+1}\right)\right\}\right) d\left(u_{n}, u_{n+1}\right) \leq \operatorname{dist}\left(f\left(u_{n}\right), r\left(u_{n+1}\right)\right) \leq d\left(w_{n}, w_{n+1}\right) .
\end{aligned}
$$

Since $\left\{t_{n}\right\}$ is increasing,

$$
\text { (iv) } \sum_{n=1}^{\infty} d\left(w_{n}, w_{n+1}\right) \leq \ell(\Gamma)<+\infty \text {. }
$$

If $\left\{d\left(u_{n}\right)\right\}$ is unbounded, define $\bar{c}(s)=c(s-1)$ for $s>1$ and select $\left\{u_{i_{k}}\right\}_{k=1}^{\infty}$ so that $i_{1}=1$ and $i_{k+1}$ is the smallest integer $j$ such that

$$
\begin{aligned}
& d\left(u_{j}\right) \leq d\left(u_{n+1}\right) \text { if } d\left(u_{i_{k}+1}\right) \leq d\left(u_{i_{k}}\right) \text {; otherwise, take } i_{k+1}=i_{k}+1 \text {. Then } \\
& \sum_{n=1}^{\infty} c\left(\max \left\{d\left(u_{n}\right), d\left(u_{n+1}\right)\right\}\right) d\left(u_{n}, u_{n+1}\right) \\
& \geq \sum_{n=1}^{\infty} c\left(d\left(u_{n}\right)\right)\left|d\left(u_{n+1}\right)-d\left(u_{n}\right)\right| \\
& 2 \sum_{k=1}^{\infty} c\left(d\left(u_{i_{k}}\right)\right)\left(d\left(u_{i_{k}+1}\right)-d\left(u_{i_{k}}\right)\right) \\
& 2 \int^{+\infty} \bar{c}(s) d s \\
& =+\infty \text {. }
\end{aligned}
$$

This contradicts $(i i i)$ and (iv). Thus $s=\sup \left\{d\left(u_{i}\right): i=1,2, \cdots\right\}<+\infty$ and (iii) implies

$$
c(s) d\left(u_{n}, u_{n+1}\right) \leq d\left(w_{n}, w_{n+1}\right), \quad n=1,2, \cdots .
$$

In conjunction with (iv), the above in turn implies that $\left\{u_{n}\right\}$ is a Cauchy sequence. Since $X$ is complete, $u_{n} \rightarrow x \in \bar{D}$. Moreover, since $r\left(u_{n}\right)=2 d\left(u_{n}, u_{n+1}\right) \rightarrow 0$, it follows that $x$ is not in $D$. Also, since $t_{n} \uparrow t \in[0,1], w_{n} \rightarrow^{*}=\Gamma(t)$, and the assumption that $f$ is closed implies $w^{*} \in f(x)$. To complete the proof, observe that

$$
\begin{aligned}
\operatorname{cr}\left(x_{0}\right) / 2 & \leq c\left(\max \left\{d\left(u_{1}\right), d\left(u_{2}\right)\right\}\right) d\left(u_{1}, u_{2}\right) \\
& \leq \operatorname{dist}\left(f\left(u_{1}\right), f\left(u_{2}\right)\right) \\
& \leq d\left(w_{1}, w_{2}\right)
\end{aligned}
$$

Therefore,

$$
\begin{aligned}
\inf \{\ell(w, y): w \in f(x)\} & \leq \ell\left(w^{*}, y\right) \\
& \leq \ell(\Gamma)-d\left(w_{1}, w_{2}\right) \\
& \leq \inf \left\{\ell(w, y): w \in f\left(w_{0}\right)\right\}+\epsilon-d\left(w_{1}, w_{2}\right)
\end{aligned}
$$




$$
\begin{aligned}
& \leq \inf \left\{l(w, y): w \in f\left(x_{0}\right)\right\}-\operatorname{cr}\left(x_{0}\right) / 4 \\
& <\inf \left\{l(w, y): w \in f\left(w_{0}\right)\right\},
\end{aligned}
$$

and, since $x \in x \backslash D$, this contradicts (b).

The final assertion of the theorem follows from the fact that, if $D=X$, then

(b) is satisfied vacuously.

\section{APPLICATIONS TO ACCRETIVE MAPPINGS}

Let $X$ be a real Banach space and $D \subset X$. We recall that a mapping $A: X \rightarrow 2^{X}$ is said to be accretive if for each $x, y \in D, u \in A(x), \quad v \in A(y)$ :

$$
\langle u-v, x-y\rangle_{+} \equiv \sup \{\langle u-v, j\rangle: j \in J(x, y)\} \geq 0 .
$$

Since the unit ball of $\mathrm{x}^{*}$ is weak* compact, the above supremum is attained and thus, by Lemma 1.1 of Kato [5], $\langle u-v, x-y\rangle_{+} \geq 0$ iff for each $\lambda \geq 0$,

$$
\|\mathrm{x}-\mathrm{y}\| \leq|(\mathrm{x}-\mathrm{y})+\lambda(\mathrm{A}(\mathrm{u})-\mathrm{A}(\mathrm{v}))| \text {. }
$$

Therefore $A: D \rightarrow 2^{X}$ is accretive if for each $\lambda>0, J_{\lambda} \equiv(I+\lambda A)^{-1}$ is a nonexpansive mapping of $(I+\lambda A)(D)$ onto D. If $(I+\lambda A)(D)=X$ for some (hence all) $\lambda>0$, then $A$ is said to be m-accretive.

Finally, A:D $\rightarrow 2^{X}$ is said to be strongly accretive if A-cI is accretive for some $c>0$.

For our first application we require the following version of Deimling's domain invariance theorem of [6]. Schöneberg's modification (see [7]) of the Crandall-Pazy proof ([8]) of this result carries over from point-valued mappings to set-valued mappings without essential change.

THEOREM 3.1 (cf. [7]). Let $\mathrm{X}$ be a Banach space, $\mathcal{B}(\mathrm{X}$ ) the nonempty bounded closed subsets of $\mathrm{X}$, and $\mathrm{H}$ the Hausdorff metric on $B(\mathrm{X})$. Suppose $\mathrm{U} \subset \mathrm{X}$ is open, and let $T: U \rightarrow B(X)$ be continuous (relative to $H$ ) and satisfy for some c > 0 ,

(i) $|\mathrm{T}(\mathrm{x})-\mathrm{T}(\mathrm{y})| \geq c\|\mathrm{x}-\mathrm{y}\|$;

(ii) the mapping $\mathrm{R}: \mathrm{U} \rightarrow \mathcal{B}(\mathrm{X})$ defined for fixed $\mathrm{y}_{0} \in \mathrm{X}$ by

$$
\begin{aligned}
R(x)= & c^{-1}\left(T(x)-y_{0}\right)-x \quad(x \in U) \quad \text { satisfies } \\
& \|u-v\| \leq|(u-v)+t(R(u)-R(v))|(u, v \in U, t \geq 0) .
\end{aligned}
$$

Then $T(U)$ is an open subset of $X$.

This theorem can be proved as follows. Let $x_{1} \in U$ and $y_{1} \in T\left(x_{1}\right)$. Choose 
$r>0$ and $\rho>0$ so that $B\left(x_{1} ; r+\rho\right) \subset U$. Fix $y \in B\left(y_{1} ; c r\right)$ and define $R: U \rightarrow B(X)$ as in (ii). It must be shown that there exists $x \in U$ such that $0 \in \mathrm{x}+\mathrm{R}(\mathrm{x})$; thus, $0 \in \mathrm{T}(\mathrm{x})-\mathrm{y}$ and $\mathrm{y} \in \mathrm{T}(\mathrm{x})$, from which $\mathrm{B}\left(\mathrm{y}_{1} ; \mathrm{cr}\right) \subset \mathrm{T}(\mathrm{U})$. Let $\psi:[0,1] \rightarrow[0,1)$ satisfy $\int_{1}^{\infty} \psi(s) d s \leq \rho$. For $u \in U, v \in R(u)$ and $\Sigma>0$, let

$$
\Lambda(\mathrm{u}, \mathrm{v}, \Sigma)=\{\mathrm{c} \in[0,1]:(1-\mathrm{c}) \mathrm{u}-\mathrm{cv} \in \mathrm{U} \text { and } \mathrm{H}(\mathrm{R}((1-\mathrm{c}) \mathrm{u}-\mathrm{cv}), \mathrm{R}(\mathrm{u}))<\psi(\Sigma+1)\},
$$

and let $\lambda(u, v, \Sigma)=\sup \Lambda(u, v, \Sigma)$. (Since $U$ is open and $R$ continuous, $\Lambda(\mathrm{u}, \mathrm{v}, \Sigma) \neq \varnothing$.

Now let $c_{1}=1$ and $v_{1}=c^{-1}\left(y_{1}-y\right)-x_{1}$, and select $c_{2} \in \Lambda\left(x_{1}, v_{1}, 1\right)$ so that $2 c_{2} \geq \lambda\left(x_{1}, v_{1}, 1\right)$. Next, select $v_{2} \in R\left(\left(1-c_{2}\right) x_{1}-c_{2} v_{1}\right)$ so that $\left\|v_{2}-v_{1}\right\|<\psi\left(c_{1}+1\right)$, and define $\left\{x_{n}\right\},\left\{c_{n}\right\}$, and $\left\{v_{n}\right\}$ recursively by taking

$$
x_{n+1}=\left(1-c_{n+1}\right) x_{n}-c_{n+1} v_{n} \text {, }
$$

where $c_{n+1} \in \Lambda\left(x_{n}, v_{n}, \sum_{j=1}^{n} c_{j}\right)$ is chosen so that $2 c_{n+1} \geq \lambda\left(x_{n}, v_{n}, \sum_{j=1}^{n} c_{j}\right)$, and then select $v_{n+1} \in R\left(x_{n+1}\right)$ so that

$$
\left\|v_{n+1}-v_{n}\right\| \leq \psi\left(1+\sum_{j=1}^{n} c_{j}\right) .
$$

From this point on it is possible, except for obvious modifications (generally, replacing $R\left(x_{i}\right)$ with $\left.v_{i}\right)$, to follow Schöneberg's proof and obtain a point $x \in U$ for which $|x+R(x)|=0$. Since $R(x)$ is closed, $0 \in x+R(x)$. We refer to [7] for the details.

We now prove the analog of Theorem 3 of [1].

THEOREM 3.2. Let $X$ be a Banach space with $D$ an open subset of $X$, let $c:[0, \infty) \rightarrow[0, \infty)$ be a continuous nonincreasing function for which $\int^{+\infty} c(s) d s=+\infty$, and suppose $T: \bar{D} \rightarrow B(X)$ is continuous on $\bar{D}$ and locally strongly accretive on $D$ in the following sense: Each point $z \in D$ has a neighborhood $N$ such that for each $x, y \in N$, if $u \in T(x)$ and $v \in T(y)$, then for some $j \in J(x, y)$,

$$
\langle u-v, j\rangle \geq c(\max \{\|x\|,\|y\|\})\|x-y\|^{2} \text {. }
$$

Then the following are equivalent:

(a') $0 \in T(D)$.

$\left(b^{\prime}\right)$ There exists $x_{0} \in D$ such that $\left|T\left(x_{0}\right)\right| \leq|T(x)|$ for each $x \in \partial D$. PROOF. Let $z \in D$ and let $N$ be a bounded neighborhood of $z$ for which (*) 
holds for all $x, y \in N$. Then the assumptions on $c \operatorname{imply} c=\inf \{c(\|u\|): u \in N\}>0$. If $u \in T(x)$ and $v \in T(y)$ for $x, y \in N$, for suitable $j \in J(x, y)$,

$$
\langle u-v-\underline{c}(x-y), j\rangle \geq 0 \text {. }
$$

Thus, by Lemma 1.1 of Kato [5], for each $\lambda \geq 0$,

$$
\|x-y+\lambda((u-v)-\underline{c}(x-y))\| \geq\|x-y\|
$$

and since this is true for all $u \in T(x)$ and $v \in T(y)$,

$$
\mid x-y+\lambda(T(x)-T(y))-\underline{c}(x-y)) \mid \geq\|x-y\|(x, y \in N, \lambda \geq 0) .
$$

Taking $\lambda=\underline{c}^{-1}$ in the above,

$$
|\mathrm{T}(\mathrm{x})-\mathrm{T}(\mathrm{y})| \geq \underline{c}\|\mathrm{x}-\mathrm{y}\| \quad(\mathrm{x}, \mathrm{y} \in \mathrm{N}) .
$$

Also, if $R: N \rightarrow B(X)$ is defined by $R(x)=c^{-1}\left(T(x)-y_{0}\right)-x$ (for fixed $\left.y_{0} \in X\right)$, then $T(x)-T(y)=\underline{c}(R(x)-R(y))+\underline{c}(x-y)$, and it follows that for each $t z 0$,

$$
|x-y+t(R(x)-R(y))| \geq\|x-y\|(x, y \in N) \text {. }
$$

Therefore, by Theorem 3.1, T maps open subsets of $N$ (hence open subsets of $D$ ) onto open sets in $X$. Since $(*)$ implies

$$
|\mathrm{T}(\mathrm{x})-\mathrm{T}(\mathrm{y})| \geq \mathrm{c}(\max \{\|\mathrm{x}\|,\|\mathrm{y}\|\})\|\mathrm{x}-\mathrm{y}\| \quad(\mathrm{x}, \mathrm{y} \in \mathrm{N}),
$$

and since $\left(b^{\prime}\right)$ implies that $(b)$ of 2.1 holds for $y=0$, we conclude: $\left(b^{\prime}\right) \Rightarrow\left(a^{\prime}\right)$. The reverse implication is obvious.

Our second application involves the so-called $\phi$-accretive mappings ([4]). Let $\mathrm{X}$ and $\mathrm{Y}$ be Banach spaces and $\phi$ a mapping of $\mathrm{X}$ onto a dense subset of $\mathrm{Y}^{*}$ which satisfies

$$
\|\phi(x)\| \leq\|x\| \text { and } \phi(\xi x)=\xi \phi(x) \quad(x \in X, \xi \geq 0) \text {. }
$$

THEOREM 3.3. Let $X$ and $Y$ be Banach spaces and suppose $Y$ has an equivalent Fréchet differentiable norm with respect to which $Y^{*}$ is strictly convex. Let $\phi: X \rightarrow Y^{*}$ be as above, let $c:[0, \infty) \rightarrow[0, \infty)$ be a continuous nonincreasing function for which $\int^{+\infty} c(x) d x=+\infty$, and suppose $T: X \rightarrow C(Y)$ is locally lipschitzian and satisfies: For each $z \in X$ there is a neighborhood $N=N(z)$ such that for each $x, y \in N$ and each $u \in T(x), v \in T(y)$,

$$
\langle u-v, \phi(x-y)\rangle \geq c(\max \{\|x\|,\|y\|\})\|x-y\|^{2} \text {. }
$$

Then for each open set $D \subset X$ the following are equivalent:

( $\left.\mathrm{a}^{\prime \prime}\right) \quad 0 \in \mathrm{T}(\mathrm{D})$.

$\left(b^{\prime \prime}\right)$ There exists $x_{0} \in D$ such that $\left|T\left(x_{0}\right)\right| \leq|T(x)|$ for each $x \in \partial D$. 
PROOF. Since $\langle u-v, \phi(x-y)\rangle \leq\|u-v\|\|\phi(x-y)\| \leq\|u-v\|\|x-y\|$, condition (**) implles that

$$
|T(x)-T(y)| \geq c(\max \{\|x\|,\|y\|\})\|x-y\| \quad(x, y \in N) .
$$

Also the local $\phi$-accretive assumption on $T$ of Theorem 3.3 implies that $T$ is locally strongly $\phi$-accretive in the sense of Definition 2.1 of Downing and Ray. Thus by Theorem 2.1 of [9], $T$ maps open subsets of $D$ onto open sets in $Y$. The result now follows from Theorem 2.1 as in the proof of Theorem 3.2.

Our final application of the above development is a global result patterned after the approach of [10].

THEOREM 3.4. Let $X$ be a Banach space with $D \subset X$ bounded and open, let $A: D \rightarrow B(X)$ be continuous and accretive, and suppose there exists $z \in D$ such that $|A(z)|<\inf \{|A(x)|: x \in \partial D\}$.

Then there exists a (single-valued) nonexpansive mapping $f: \bar{D} \rightarrow D$ whose fixed points are zeros of $A$.

PROOF. Since $D$ is bounded, it is possible to choose $\alpha \in(0,1)$ so near 1 that $\alpha|\mathrm{A}(\mathrm{z})|+(1-\alpha)\|\mathrm{z}-\mathrm{y}\|<\inf \{\alpha|\mathrm{A}(\mathrm{x})|-(1-\alpha)\|\mathrm{x}-\mathrm{y}\|: \mathrm{x} \in \mathrm{aD}\}$

for each $y \in \bar{D}$. Fix $w \in \bar{D}$ and define $T_{w}: \bar{D} \rightarrow 2^{X}$ by

$$
T_{x}(x)=(1-\alpha)(x-w)+\alpha A(x), \quad x \in \bar{D} \text {. }
$$

Then, if $\bar{x} \in \partial D$,

$$
\begin{aligned}
\left|T_{w}(z)\right| & =|(1-\alpha)(z-w)+\alpha A(z)| \\
& \leq(1-\alpha)\|z-w\|+\alpha|A(z)| \\
& <\inf \{\alpha|A(x)|-(1-\alpha)\|x-w\|: x \in \partial D\} \\
& \leq \inf \{|\alpha A(x)+(1-\alpha)(x-w)|: x \in \partial D\} \\
& \leq\left|T_{w}(\bar{x})\right| .
\end{aligned}
$$

Also, if $u \in T_{w}(x)$ and $v \in T_{w}(y)$, then, for some $j \in J(x-y)$,

$$
\langle u-v, j\rangle \geq(1-\alpha)\|x-y\|^{2} \text {; }
$$

so by Theorem 3.2, there exists $z_{w} \in D$ such that $0 \in T_{w}\left(z_{w}\right)=(1-\alpha)\left(z_{w}-w\right)+\alpha A\left(z_{w}\right)$; i.e.,

$$
z_{w} \in w-\lambda A\left(z_{w}\right) \quad(\lambda=\alpha /(1-\alpha))
$$

By accretivity of A,

$$
\left\|z_{u}-z_{v}\right\| \leq\left|\left(z_{u}-z_{v}\right)+\lambda\left(A\left(z_{u}\right)-A\left(z_{v}\right)\right)\right| \quad(u, v \in \bar{D}) .
$$


But $z_{u} \in u-\lambda A\left(z_{u}\right)$ and $z_{v} \in v-\lambda A\left(z_{v}\right)$. Thus

$$
\left|\left(z_{u}-z_{v}\right)+\lambda\left(A\left(z_{u}\right)-A\left(z_{v}\right)\right)\right| \leq\|u-v\|,
$$

proving that the mapping $u \longmapsto z_{u}$ is nonexpansive. Finally, if $u=z_{u}$ for $u \in D$, then $u \in u-\lambda A(u)$, proving $0 \in A(u)$.

COROLIARY 3.1. Let $X$ be a Banach space for which the closed balls have the fixed point property for nonexpansive self-mappings. Suppose $A: X \rightarrow B(X)$ is continuous and accretive, and satisfies

Then $A(X)=X$.

$$
\lim _{\|x\| \rightarrow \infty}|A(x)|=+\infty
$$

PROOF. Fix $y \in X$ and define $\widetilde{A}: X \rightarrow B(X)$ by $\widetilde{A}(x)=A(x)-y$. Choose $\delta>0$ so that

$$
C=\{x \in X:|\widetilde{A}(x)| \leq \delta\} \neq \varnothing .
$$

Since $|A(x)|-\|y\| \leq|\widetilde{A}(x)| \Longrightarrow|\widetilde{A}(x)| \rightarrow \infty$ as $\|x\| \rightarrow \infty, \quad C$ is bounded, and moreover for $r>0$ sufficiently large and $x_{0} \in C$,

$$
\left|\widetilde{A}\left(x_{0}\right)\right|<\inf \{|\tilde{A}(x)|:\|x\|=r\} .
$$

Thus, $0 \in \tilde{A}(x)$ for some $x \in B_{r}(0)$; hence, $y \in A(x)$.

The analog of Corollary 3.1 for m-accretive operators is proved in [11].

\section{REMARKS.}

(1) As Torrejón observes in [3], the assumption that $c$ is nonincreasing in Theorem 2.1 (hence in Theorems 3.2, 3.3) is not really essential. To see this, define $\left\{u_{i_{k}}\right\}$ as in the proof of Theorem 2.I, fix $k \geq 1$, and use the fact that the image of $\Gamma([0,1])$ under the inverse of the restriction of $f$ to $B\left(u_{i_{k}} ; r\left(u_{i_{k}}\right) / 2\right)$ is a path.

Consequently, it is possible to obtain points $\left\{\mathrm{s}_{1}^{(\mathrm{k})}, \mathrm{s}_{2}^{(\mathrm{k})}, \ldots, \mathrm{s}_{\mathrm{n}_{\mathrm{k}}}^{(\mathrm{k})}\right\}$ in $B\left(u_{i_{k}} ; r\left(u_{i_{k}}\right) / 2\right)$ such that the numbers $d\left(u_{i_{k}}\right)=d\left(s_{1}^{(k)}\right)<d\left(s_{2}^{(k)}\right)<\cdots<d\left(s_{n_{k}}^{(k)}\right)$ $=d\left(u_{i_{k}+1}\right)$ induce a partition of $\left[d\left(u_{i_{k}}\right), d\left(u_{i_{k}}+1\right)\right]$, while at the same time $\Gamma\left(t_{i_{k}}^{(k)}\right) \in f\left(s_{i}^{(k)}\right)$ where $t_{i_{k}}=t_{l}^{(k)}<t_{2}^{(k)}<\cdots<t_{n_{k}}^{(k)}=t_{i_{k}+l}$. Moreover, if $\epsilon_{k}>0$, then the above partition may be further refined so that

$$
\sum_{i=1}^{n k-1} c\left(d\left(s_{i+1}^{(k)}\right)\right)\left(d\left(s_{i+1}^{(k)}\right)-d\left(s_{i}^{(k)}\right)\right) \geq \int_{d\left(u_{i_{k}}\right)}^{d\left(u_{i_{k}+1}\right)} c(s) d s-\epsilon_{k} \text {. }
$$


Since the left side of the above is bounded by the length of $\Gamma$ from $t_{i_{k}} t_{0} t_{i_{k}}+1$, by choosing $\left\{\epsilon_{k}\right\}$ so that $\Sigma \epsilon_{k}<\infty$, it is possible to proceed as in the proof of Theorem 2.1 to obtain (if $\left\{a\left(u_{n}\right)\right\}$ is unbounded) the contradiction:

$$
\begin{aligned}
+\infty>\ell(\Gamma) & \left.\geq \sum_{k=1}^{\infty} \sum_{i=1}^{n_{k}-1} \operatorname{dist}\left(f_{i}^{(k)}\right), f\left(s_{i+1}^{(k)}\right)\right) \\
& \geq \sum_{k=1}^{\infty} \sum_{i=1}^{n k} c\left(d\left(s_{i+1}^{(k)}\right)\right)\left(d\left(s_{i+1}^{(k)}\right)-d\left(s_{i}^{(k)}\right)\right) \\
& \geq \int^{+\infty} c(s) d s-\sum \epsilon_{k}=+\infty .
\end{aligned}
$$

(Note that this argument is merely a reworking of that of Browder [4, Theorem 4.9].)

(2) We note also that Theorem 2.1 has the following corollary (cf. [4, Theorem 4.10]).

COROLLARY. Let $X$ and $Y$ be Banach spaces, $c:[0, \infty) \rightarrow(0, \infty)$ a continuous (nonincreasing) mapping for which $\int^{+\infty} \mathrm{c}(\mathrm{s}) \mathrm{d} \mathbf{s}=+\infty$, and $\mathrm{T}: \mathrm{X} \rightarrow 2^{\mathrm{Y}}$ a closed mapping which maps open subsets of $X$ onto open sets in $Y$. Suppose each point $z \in$ has a neighborhood $N$ such that for each $x, y \in N$,

$$
|\mathrm{T}(\mathrm{x})-\mathrm{T}(\mathrm{y})| \geq \mathrm{c}(\max \{\|\mathrm{x}\|,\|\mathrm{y}\|\})\|\mathrm{x}-\mathrm{y}\| \text {. }
$$

Then $T(X)=Y$.

ACKNOWLEDGEMENT. Research supported by the National Science Foundation under grant no. MCS 80-01604-01.

\section{REFERENCES}

1. KIRK, W.A., and SCHÖNEBERG, R. Mapping Theorems for Local Expansions in Metric and Banach Spaces, J. Math. Anal. Appl. 27 (1979), 114-121.

2. RAY, W.O., and WALKER, A. Mapping Theorems for Gateaux Differentiable and Accretive Operators, Nonlinear Analysis: TMA 6 (1982), 423-433.

3. TORREJÓN, R. Some Remarks on Nonlinear Functional Equations (to appear).

4. BROWDER, F.E. Nonlinear Operators and Nonlinear Equations of Evolution in Banach Spaces, Proc. Symp. Pure Math. vol. 18, pt. 2, Amer. Math. Soc., Providence, RI, 1976.

5. KATO, T. Nonlinear Semigroups and Evolution Equations, J. Math. Soc. Japan 19 $(1967), 508-520$.

6. DEIMLING, K. Zeros of Accretive Operators, Manuscripta Math. 13 (1974), 365-374.

7. SCHÖNEBERG, R. On the Domain Invariance Theorem for Accretive Mappings, J. London Math. Soc. (2) $24(1981), 548-554$. 
8. CRANDALL, M., and PAZY, A. On the Range of Accretive Operators, Israel J. Math. $27(1977), 235-246$.

9. DOWNING, D., and RAY, W.O. Renorming and the Theory of Phi-accretive Set-valued Mappings, Pacific J. Math. (to appear).

10. KIRK, W.A., and SCHÖNEBERG, R. Some Results on Pseudocontractive Mappings, Pacific J. Math. 71 (1977), 89-100.

11. KIRK, W.A., and SCHÖNEBERG, R. Zeros of m-accretive Operators in Banach Spaces, Israel J. Math. 35 (1980), 1-8. 


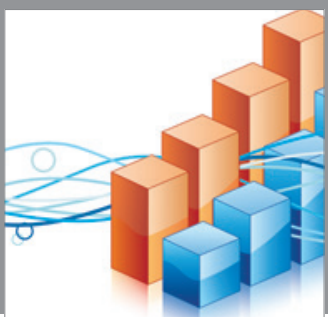

Advances in

Operations Research

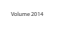

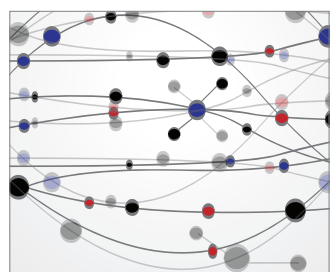

\section{The Scientific} World Journal
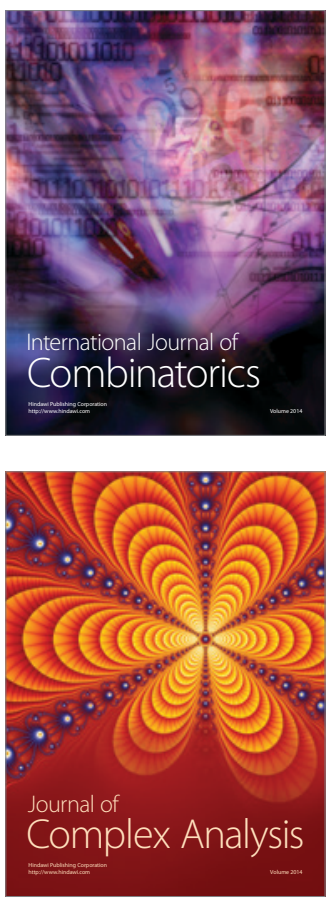

International Journal of

Mathematics and

Mathematical

Sciences
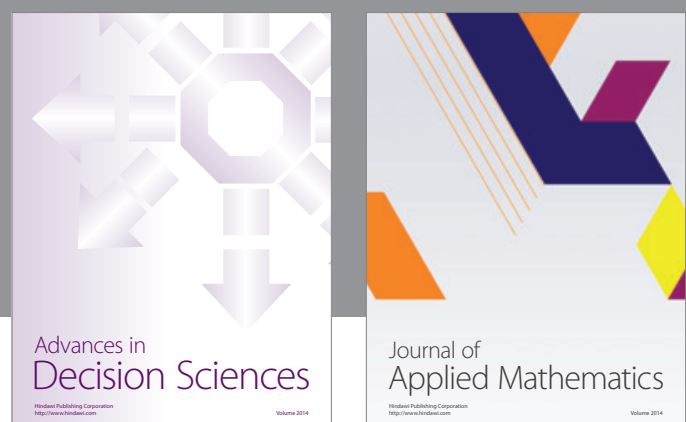

Journal of

Applied Mathematics
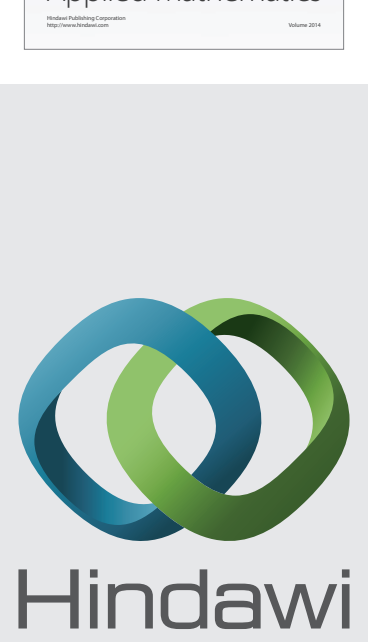

Submit your manuscripts at http://www.hindawi.com
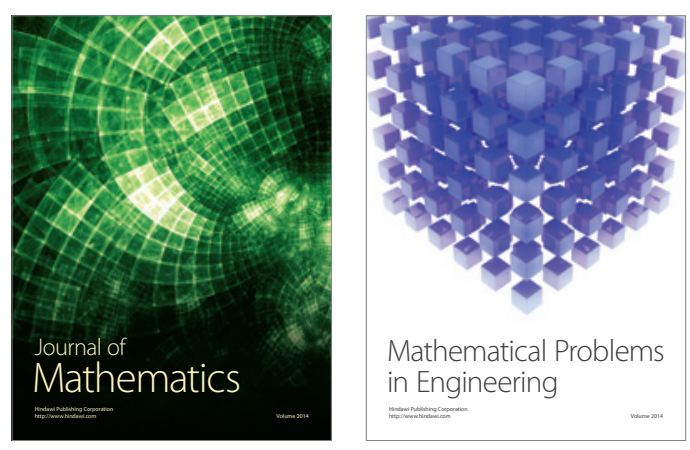

Mathematical Problems in Engineering
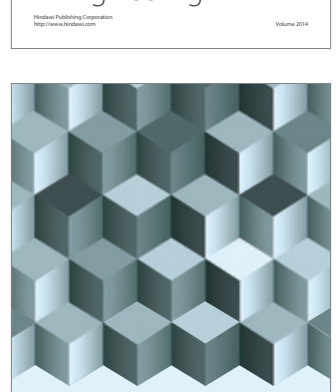

Journal of

Function Spaces
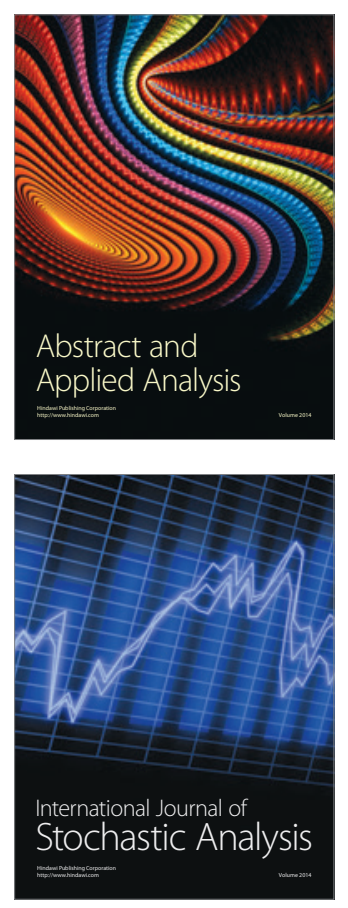

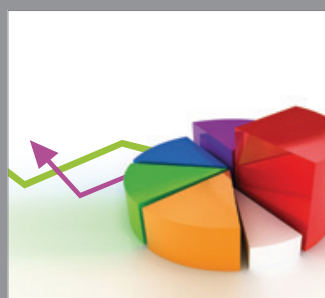

ournal of

Probability and Statistics

Promensencen
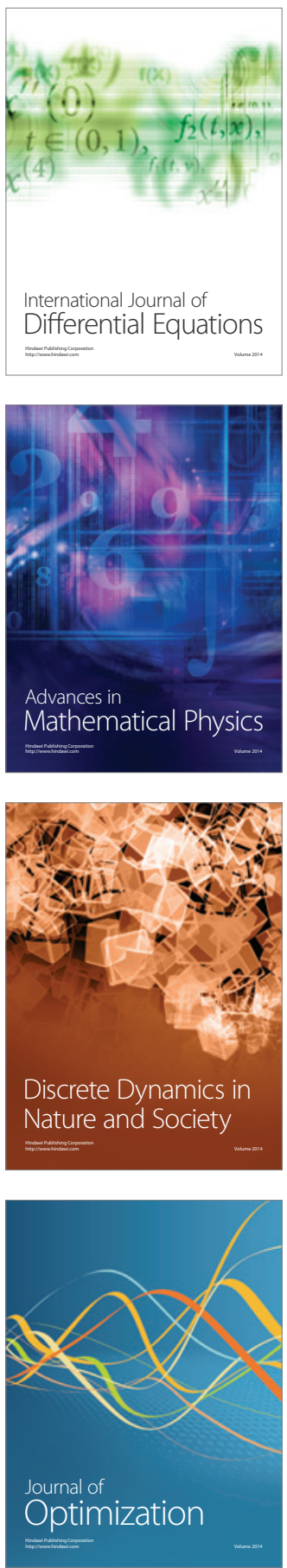